\title{
Main Microbial Genres and Its Profile of Resistance and Sensitivity to Antimicrobials Used in the Treatment of Oncological Patients with Febril Neutropenia
}

\author{
Hélvecio Cardoso Póvoa1, Richard Raphael Borges Tavares Vieira², Elias Sobreira Sathler², \\ Isabela Nardoni Bernardes'2, Rafael Batista Ferreira², Mário Henrique Couto Lima², \\ Thiago Sande Miguel2, Nayrton Kalys Cruz dos Anjos², \\ Lamara Laguardia Valente Rocha ${ }^{3}$, Daniel Almeida da Costa ${ }^{2,3}$ \\ ${ }^{1}$ Federal Fluminense University, Niterói, Brazil \\ ${ }^{2}$ The Medical School at the Center of Higher Education of Valença, Valença, Brazil \\ ${ }^{3}$ The Medical School at the Health Sciences Institute of the University Center of Caratinga, Caratinga, Brazil \\ Email: belinhax@hotmail.com,rbf_br@hotmail.com, essathler@hotmail.com, thiagosande.miguel@hotmail.com, \\ rrbtv@hotmail.com, lamara.laguardia@gmail.com, professordanielfmv@gmail.com
}

How to cite this paper: Póvoa, H.C., Vieira, R.R.B.T., Sathler, E.S., Bernardes, I.N., Ferreira, R.B., Lima, M.H.C., Miguel, T.S., dos Anjos, N.K.C., Rocha, L.L.V. and da Costa, D.A. (2018) Main Microbial Genres and Its Profile of Resistance and Sensitivity to Antimicrobials Used in the Treatment of Oncological Patients with Febril Neutropenia. Journal of Biosciences and Medicines, 6, 34-50.

https://doi.org/10.4236/jbm.2018.61005

Received: May 21, 2017

Accepted: January 7, 2018

Published: January 10, 2018

Copyright $\odot 2018$ by authors and Scientific Research Publishing Inc. This work is licensed under the Creative Commons Attribution International License (CC BY 4.0).

http://creativecommons.org/licenses/by/4.0/

c. (†) Open Access

\begin{abstract}
Objective: To determine the main microbial genotypes and their antimicrobial resistance and susceptibility profile commonly used in the treatment of oncologic patients with febrile neutropenia, carried out through the review of the clinical histories of the patients' medical records at the Hospital do Câncer de Muriaé-Fundação Cristiano Varella. Methods: Quantitative research, determined the susceptibility profile of microorganisms in patients with febrile neutropenia from April 2007 to April 2008 by reviewing clinical histories of patients' medical records at the referred hospital. Results: Of the total of 8 patients evaluated with Gram-negative microorganisms, 50\% of the patients were female and $50 \%$ were male. Of the 18 patients evaluated with Gram-positive microorganisms $66 \%$ were female and $34 \%$ male. Gram-positive bacteria are prevalent and cause around $60 \%$ of documented bacteremias, although Gram-negative bacteria are more common in febrile neutropenic patientes. Conclusion: Exaggerated use of antimicrobials in hospitalized patients leads to the suppression of drug-sensitive microorganisms from the intestinal flora and promotes the persistence and growth of resistant bacteria. The antibiotic should be used in a curative manner with other therapeutic measures with the determined bacterial infection.
\end{abstract}




\section{Keywords}

Febrile Neutropenia Antimicrobial Resistance, Oncologic Patients

\section{Introduction}

The main factors that increase environmental carcinogens or allow for a longer and longer exposure of humans to these agents, thus contributing to the increase in the incidence of cancer, are the urbanization, industrialization and life expectancy of the population [1].

Cancer is one of the causes of increased mortality and morbidity in the world, with more than ten million new cases and more than six million deaths per year. The cancer-related mortality rate corresponds to $15 \%$, and its rates are increasing and high, probably because the disease is diagnosed in advanced stages. The number of cancer cases has increased considerably, especially since the last century, and is now one of the most important public health problems in the world. In these circumstances, it has already been considered an acute and invariably fatal disease, and is one of the main causes of death in Brazil [2].

Today it is considered a chronic disease, with prospects of cure in most cases. Thus, 70\% of people affected by cancer can be cured, when the diagnosis occurs early and treatment is performed in specialized centers. Diagnosis is a stressful time and the chemotherapy protocols used in the treatment, although they offer a variety of medical care, do not contemplate the patient and his/her relatives in the discovery and confrontation of the diagnosis, causing pain to the patient [3].

The objective of this work is to determine the main microbial genotypes and their antimicrobial resistance and susceptibility profile commonly used in the treatment of oncologic patients with febrile neutropenia, carried out through the review of the clinical histories of the patients' medical records at the Hospital do Câncer de Muriaé-Fundação Cristiano Varella.

\subsection{Immune System}

The immune system is a system of defense of the organism, with capacity to fight invading microorganisms, to remove dead cells, to renew certain structures, to reject grafts and immune memory. It is also active against altered cells, which arise daily in the body as a result of abnormal mitoses. These cells, if not destroyed, can give rise to tumors [4].

This defense capability is operationalized by primary immune lymphoid organs, when cells specialized in promoting immune response in the presence of non-self antigens are developed. The immune system also maintains the memory of the first contact, so that, in a second exposure to the same external agent, there is induction of a more pronounced response. The innate immunity, responsible for the initial immune response, involves physical barriers, enzymes, complements and cytokines in its humoral component, and neutrophils, basophils, 
eosinophils, macrophages and natural killer (NK) cells in its cellular component. The humoral adaptive immune response operates by antibodies produced by $\mathrm{B}$ lymphocytes and cellular adaptive immunity is mediated by $\mathrm{T}$ lymphocytes (suppressors or inducers). Accessory and effector cells capable of destroying non-self agents can also be found within phagocytes or even in other cells of the immune system [5].

\subsection{Immune System Cells}

The cells of the immune system are called leukocytes because they are white blood cells. The number of leukocytes per milliliter of blood in the normal adult is 5000 to 10,000 . At birth, the child's blood contains 20,000 leukocytes $/ \mathrm{mm}^{3}$ of blood and decreases with life and at 12 years reaches the adult range. This is because the child does not yet have the natural barriers of the organism completely developed, having more facilities to contract infections of diverse natures. For this reason it is necessary that there is a larger leukocyte population for the protection of the child [6].

\subsection{Neutrophils}

Neutrophils are blood-derived cells originating from a reservoir of bone marrow precursor cells through serial divisions and synchronous stages of maturation. The rate of neutrophil production is surprisingly high. These cells normally play their role in the body's phagocytic defense as long as its blood count is above $1000 / \mathrm{mm}^{3}[7]$.

They are the most populous leucocytes in the blood, being part of approximately $65 \%$ of the leukocytes of the blood, are the main phagocytes and participate in the inflammatory reaction, being sensitive to chemotactic agents released by mast cells, basophils and complement [8].

Neutrophil leukocytosis may indicate a bacterial infection, they participate in phagocytosis of bacteria and are highly stimulated in an infection of this type, called left shunt in the blood count study, which is an examination that analyzes the quantitative and morphological variations of the Blood [8].

\subsection{Fever}

Fever is the controlled elevation of body temperature above normal values for the individual. Normal axillary temperature ranges from $36.5^{\circ} \mathrm{C}$ in the morning to $37.2^{\circ} \mathrm{C}$ in the afternoon; The buccal temperature is approximately $0.5^{\circ} \mathrm{C}$ more than the axillary, and the rectal temperature is $0.8^{\circ} \mathrm{C}$ to $1^{\circ} \mathrm{C}$ higher than the axillary, that is, $37.8^{\circ} \mathrm{C}$, and can reach up to $38.5^{\circ} \mathrm{C}$ [9].

However, fever may be defined as a record of axillary temperature greater than $38.5^{\circ} \mathrm{C}$ or of records greater than $38.1^{\circ} \mathrm{C}$ in a period of 12 to 24 hours [7].

\subsection{Febrile Neutropenia}

Neutropenia occurs when neutrophil counts decrease to values below $1000 / \mathrm{mm}^{3}$ 
in the blood, being an important risk factor in the immunocompromised patient. Neutropenic patients with fever are more than or equal to $60 \%$ likely to develop infection when the neutrophil count is below $100 / \mathrm{mm}^{3}$ and a $20 \%$ chance of developing bacteremia. There is a direct relationship between the number of neutrophils in the peripheral blood and the occurrence of infection [7].

Febrile neutropenia is an infectious emergency in oncology patients. Both solid tumors and neoplasms with infiltrations in the bone marrow predispose to this type of complication. It may be defined as febrile episodes associated with a neutrophil count below $1000 / \mathrm{mm}^{3}$ with a predicted decline of $500 / \mathrm{mm}^{3}$ or less [10].

The clinical evaluation of the febrile neutropenic patient should focus on potential sites of infection and possible causal agents, assessing the patient's risk of developing infection [7].

Most episodes of febrile neutropenia are treated empirically without identification of the site or etiologic agent, such as fever of undetermined origin. In the other cases of documented infection, several variables are involved in the etiology. Factors such as recent hospital admission, previous use of prophylactic or therapeutic antimicrobial agents, baseline disease, intensity and time of neutropenia, use of long-term central venous catheters or other invasive devices should be considered in the determination of initial empirical therapy. The sites most commonly involved are pulmonary infections, catheter-related bloodstream infections, skin and soft tissue infections, among others. In general, bacterial agents are mainly responsible for infection during febrile neutropenia [11].

\subsection{Antimicrobials}

Antimicrobials account for approximately $12 \%$ of all outpatient prescriptions, suggesting an estimated $\$ 15$ billion a year in these drugs. The unrestrained use of antibiotics without careful evaluation of their appropriate indications may lead to the growth of resistant strains, that is, to cause a selective mutation of microorganisms [12].

The pathogenesis of bacterial infection comprises the beginning of the infectious process. Bacteria characterize by their capacity of transmission, adhesion and invasion of cells and tissues, toxigenicity and ability to escape from the immune system of the host. Disease occurs if the bacterium or the immunological reactions present to it harm the host [13].

Among the bacteria, we can highlight the Staphylococcus that frequently hemolysate the blood, coagulate the plasma and produce a variety of enzymes and extracellular toxins. Staphylococci rapidly develop resistance to antimicrobials, thus causing therapeutic problems. In infections, there is a localized and painful inflammatory reaction, which undergoes central suppuration and heals rapidly when the pus is drained [14].

Enterobacteriaceae constitute a large heterogeneous group of Gram-negative bacteria whose natural habitat is the intestinal tract of humans and animals. 
Among the main genera is Escherichia coli. In the hospital setting these bacteria are commonly transmitted by instrumentation or by parenteral medications. Its control depends on hand washing, rigorous asepsis, sterilization of equipment, disinfection, restriction of intravenous therapy and sterile urinary tract [13].

\subsection{Sensitivity and Resistance to Antimicrobials}

Patients exposed to previous antibiotic use have a higher incidence of non-susceptible strains than patients who have not previously received antibiotics. Each year, in the United States, 160 million antibiotic prescriptions are filled, corresponding to 22.7 million $\mathrm{kg}$, 50\% for use in humans and $50 \%$ for use in animals, agriculture and hydroculture. In a population of 275 million, this amounts annually to 30 prescriptions per 100 people and $4.1 \mathrm{~kg}$ of antibiotics per 100 people [15].

Although antimicrobial resistance has increased in the last years, there is great disparity between the different sites where the studies were carried out, and high and low rates of penicillin resistance have been described in nearby geographic regions. This is a very worrying situation, considering the great ease of transportation in the present day, where the traffic of people between different localities can quickly contribute to the dissemination of resistant strains [14].

The erroneous antibiotic-therapeutic treatment is a factor of contribution of infections that is more discussed at the present time, since it is responsible for the development of bacterial resistance, as a consequence of the bacterium acquiring resistance, being the lack of options to be used as treatment in last cases, And the fact that the onset of bacterial resistance is very fast compared to the process of developing new drugs. Under these conditions, the costs of treatment against these bacteria in the health system and in the hospitals themselves are more pronounced [16].

The resistance of bacteria is a result of how each medical community uses antimicrobials. For this reason, the characterization of the bacterial incidence and its resistance profile is the basis for directed and adequate antibiotic therapy [16].

\subsection{Susceptibility in Oncologic Patients}

Polychemotherapy, i.e., use of more than one cytotoxic agent in combination, is able to slow down the tumor growth mechanism, enabling better treatment responses. Its main advantages are the additive effect that is produced; The potentiation of the therapeutic effect of one drug with the use of another; Delay of tumor resistance; Possibilities of lower doses and, consequently, decrease of toxic and collateral effects [1].

The cancer patient presents with great laughter of infection due to being immunocompromised organism, undergoing treatment with chemotherapy, radiotherapy or undergoing surgery. Also, the use of invasive procedures can contribute significantly to the occurrence of infection, justifying the existence of 
several infectious topographies such as respiratory, urinary, bloodstream, surgical, cutaneous and gastrointestinal areas [17].

As a result of cancer, suppression of the bone marrow occurs, verifying a decrease in the number of functional granulocytes. When the granulocyte count falls to less than 500 neutrophils per microliter, the patient becomes highly susceptible to opportunistic infections by bacteria. The antibacterial defenses of this patient may be reinforced by supplies of certain important cytokines in hematopoiesis, such as colony stimulating factors [13].

In cancer patients, risk factors may overlap, since, in addition to the immunocompromising caused by the pathology itself, they are submitted to surgical, chemotherapeutic and/or radiotherapeutic treatment, and neutropenia, induced by chemotherapy, is one of the greatest risks for infection in this population [18].

Most antineoplastic chemotherapeutics act in a non-specific way, damaging both malignant and benign cells, especially those of rapid division, especially hematopoietic, germ, hair follicle and lining epithelium of the gastrointestinal system. Thus, the main side effects are neutropenia, thrombocytopenia, anemia, nausea and vomiting, mucositis, in addition to cardiac, hepatic and renal toxicities. Neutropenia increases the risk of developing infection, which is enhanced by the patient's exposure to the hospital environment, invasive procedures and length of stay [19].

Surveillance culture has been singled out as the most sensitive approach for identifying colonized patients. Some studies recommend its performance in all patients admitted to the hospital, independently of the evaluation of the risk criteria. However, the time spent to perform the microbiological tests and the high cost of the procedure have made it difficult to implement this routine in most hospitals, and the clinical criteria are the most promising and cost-effective option when compared to the surveillance culture [18].

The present work aims to determine the main microbial genotypes and their antimicrobial susceptibility and resistance profile commonly used in the treatment of oncologic patients with febrile neutropenia, carried out through the review of the clinical histories of patients' medical cancer records of Muriaé, Brazil.

\section{Methodology}

We chose quantitative research in the case of an observational, descriptive, retrospective study. The study was conducted at the Hospital do Câncer de Muriaé-Fundação Cristiano Varella, located in the city of Muriaé, Brazil.

The susceptibility profile of the microorganisms in patients with febrile neutropenia from April 2007 to April 2008 was determined. The identification of the patients was performed by the records of reports in the clinical analysis laboratory.

Data were collected in the medical records of all febrile neutropenic patients at the Hospital do Câncer de Muriaé from April 2007 to April 2008. 
The source of this data was the review of the clinical histories of patients' records in the files of the referred hospital.

\section{Results and Discussion}

Data were collected in the medical records of all febrile neutropenic patients at the Hospital do Câncer de Muriaé from April 2007 to April 2008.

Of the 8 patients evaluated with Gram-negative microorganisms, 50\% of the patients were female and $50 \%$ were male. Of the 18 patients evaluated with Gram-positive microorganisms 66\% were female and 34\% male.

As the objective of the study was to analyze all febrile neutropenic patients, there was no criterion for sex selection, that is, all hospitalized from April 2007 to April 2008 with the disease in question were evaluated. As can be seen in Figure 1 , there was a predominance in women, it does not interfere in the results obtained, since febrile neutropenia affects both men and women [7].

According to the results presented in Figure 2, it is possible to observe the female predominance among the patients with Gram positive microorganisms, and the same proportion in patients with Gram negative microorganisms, of the total evaluated patients.

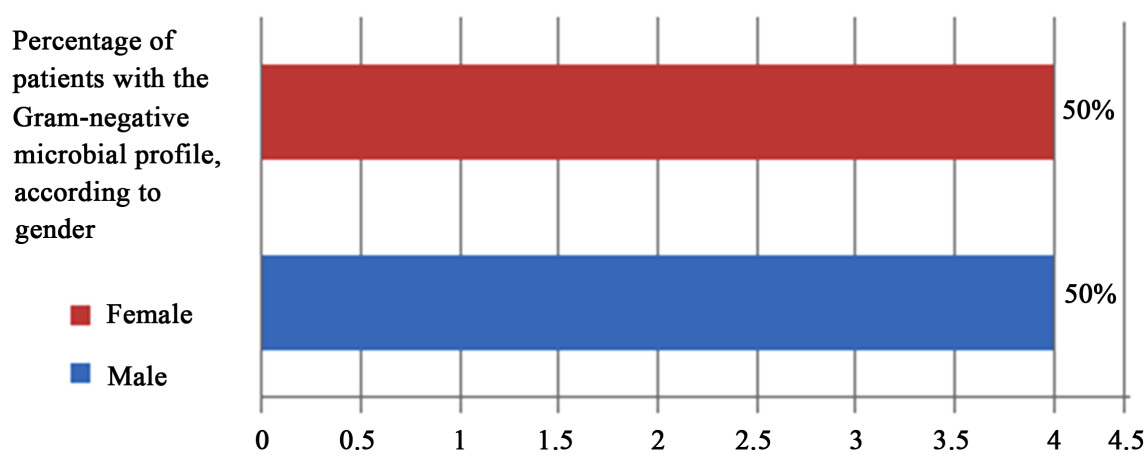

Figure 1. Distribution of febrile neutropenic patients with the microbial profile Gram negative, according to sex.
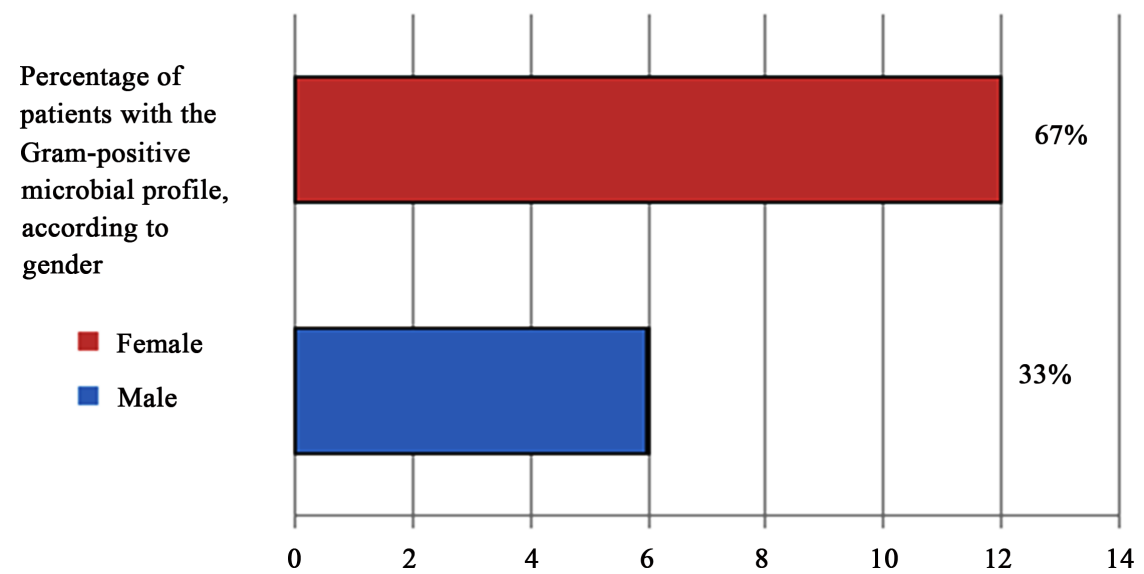

Figure 2. Distribution of febrile neutropenic patients with the Gram-positive microbial profile, according to gender. 
Figure 3 shows the distribution of the patients according to the microorganisms, properly grouped. The pattern of infectious agents has changed significantly over time. Gram-negative bacteria were the most common pathogens in febrile neutropenic patients and Gram-positive bacteria are currently prevalent and cause around $60 \%$ of documented bacteremias [10].

From the analysis of Figure 3, we can see the distribution of microorganisms with predominance of Gram positive bacteria in $69 \%$ of patients, and only $31 \%$ of Gram negative bacteria.

The more intensive chemotherapeutic treatment associated with a more severe mucositis or diarrhea leads to greater damage to the mucosal barrier causing infections by Gram positive bacteria of the normal flora, such an affirmation can be an explanation for the modification. Cancer patients receive a central venous catheter that can easily be colonized with skin bacteria and are also a well-known risk factor for infection by Gram-positive bacteria. The use of more active antibiotics against Gram negative than Gram positive may also play a role in changing this microbiological pattern [13].

The bacteria that commonly cause disease in immunocompromised patients are the same ones that affect immunocompetent individuals; gram-positive patients account for $60 \%$ to $70 \%$ of infections episodes, documented microbiologically in neutropenic patients, and the most common microorganisms are coagulase negative staphylococci and enterococci Resistant to vancomycin [18].

Of the patients analyzed in this study, with a Gram-negative microbial profile, 75\% had Escherichia coli infection and 25\% had Acinetobacter sp. Infection.

Figure 4 can be explained because the species Escherichia coli is a type of enterobacteria, being part of the normal flora of the human intestine and accidentally causes diseases when bacteremia occurs. They are opportunistic enteric

Percentage of patients according to the microbial profile.

Gram-negative

Gram-positive

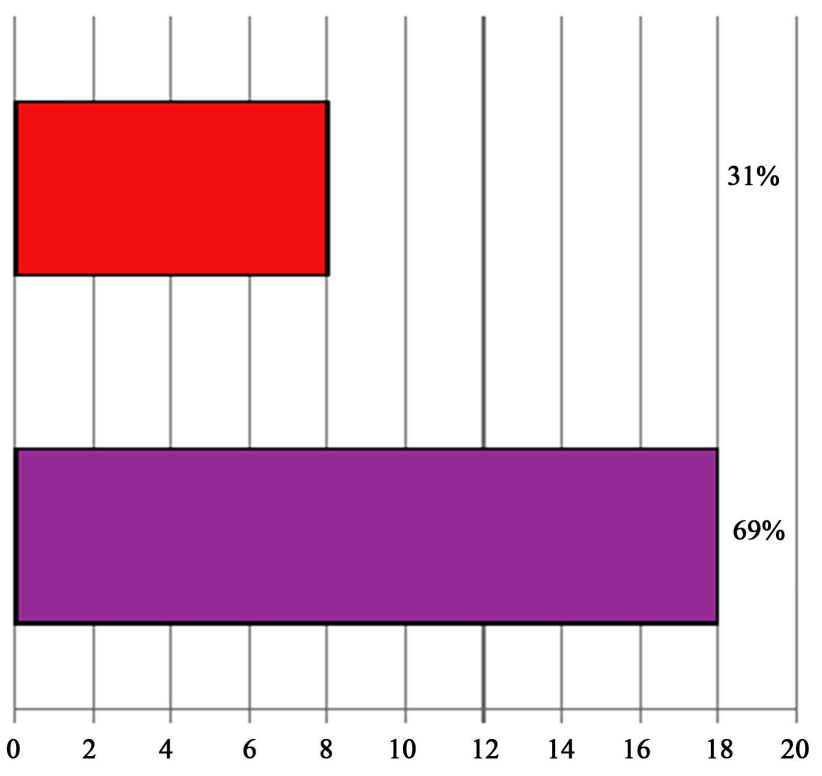

Figure 3. Distribution of febrile neutropenic patients according to the microbial profile. 


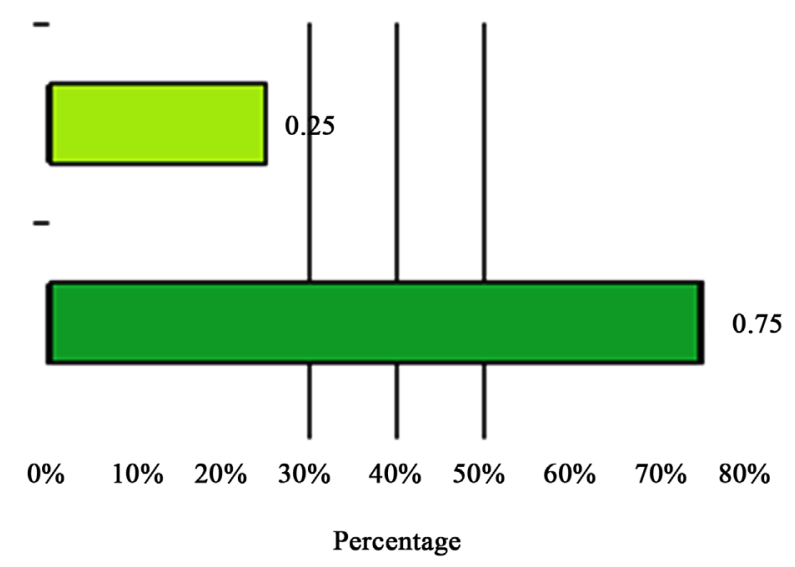

Figure 4. Distribution of febrile neutropenic patients according to the type of microorganism with a Gram negative profile.

bacteria, causing diseases when introduced into debilitated patients. In the hospital environment they are transmitted by the health team, by unsterilized instruments or by parenteral medications. Acinetobacter bacteria species are more uncommon, being a hospital pathogen of low virulence, which can be found in blood cultures of patients with intravenous plastic catheters, considering that this is almost always the source of infection [10].

Of the patients analyzed in this study, with a Gram positive microbial profile, $56 \%$ presented infection by Sthapylococcus epidermidis, 22\% Sthapylococcus aureus, 16\% Enterococcus and 5\% Rhodococcus sp.

The analysis of Figure 5 is explained because Staphylococcus is present in the normal microbiota of the skin and mucosa of humans. Almost all individuals suffer some type of infection during life, whose severity ranges from a food poisoning that is of little importance until serious and potentially fatal infections. The spread of contact infection takes on greater importance in hospitals where a large proportion of staff and patients harbor antibiotic-resistant Staphylococcus in the nose or skin. Such a bacterium has few methods to prevent its widespread dissemination from the carriers. In hospitals, areas such as: nurseries, intensive care unit, surgical center and cancer chemotherapy wards are at greater risk, which may result in severe clinical disease [13].

Enterococcus is among the most common causes of nosocomial infections, particularly in intensive care units, and is selected as a result of antibiotics to which they are resistant. Enterococcus is transmitted from one patient to another, primarily through the hands of the healthcare team, some of whom may harbor Enterococcus in the gastrointestinal tract. At times they can be transmitted through medical devices. An important problem related to Enterococcus is the marked resistance to antibiotics. The Rhodococcus genus occasionally causes infections such as pneumonia in immunosuppressed patients with abnormal cell immunity. It is found in the soil and manure of cattle, swine and sheep, rarely causing diseases and infections in humans, being explained the reason for the low prevalence in the result of the research in question [13]. 

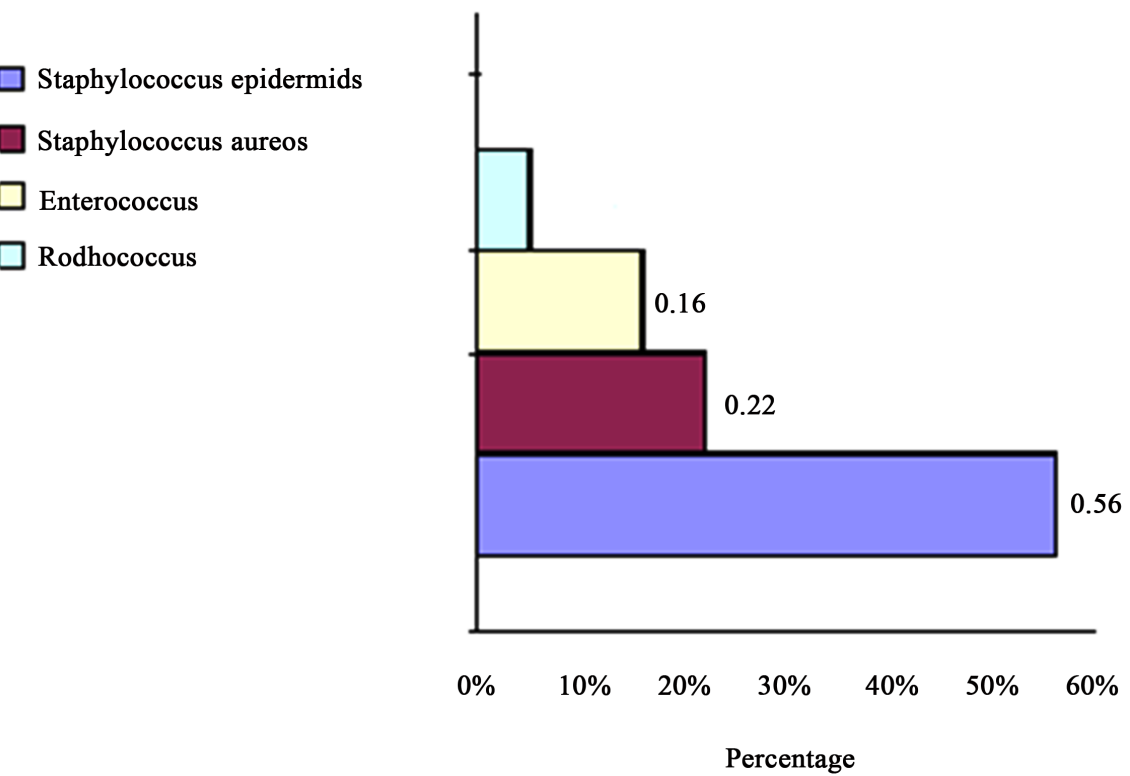

Figure 5. Distribution of febrile neutropenic patients according to the type of microorganism with Gram positive profile.

The prevalence of infections and the consequent consumption of the drugs to treat them lead to many prescription errors related to uncertainty of diagnosis and pharmacological ignorance. It is common not to recognize that antimicrobials are specific drugs and therefore only effective for certain infectious agents. The availability of antimicrobials, accompanied by poor publicity, accentuates abusive use. The development of resistance is a natural biological phenomenon that followed the introduction of antimicrobial agents in clinical practice. Resistance rates vary locally depending on local consumption of antimicrobials. Bacterial resistance is a worldwide concern and is the subject of the most current publications on antimicrobials. These are the only drugs that influence not only the patient being treated, but the whole ecosystem where he is inserted, with profound potential repercussions [17].

The control of microbial resistance depends on factors such as: development of new drugs, development of vaccines, detection of microbial resistance profile in hospitals, implementation of hospital infection control measures, development of therapeutic protocols for prevalent infections, training of undergraduate students in health areas in the diagnosis and management of common infections, educational programs for users to prevent infection and reduce transmission, maintenance of the quality of laboratories for microbiological analysis, promotion of the rational use of antimicrobials, dispensing of antibiotics with medical prescription only and marketing authorization only for antibiotics meeting international standards of efficacy, safety and quality [20].

Some studies have been carried out in order to characterize the resistance and to establish risk factors for its occurrence. The phenomenon is complex and has multiple causes, some already well determined, others still to be clarified, well known in hospital settings. Among the strategies adopted for the management of 
this serious public health problem, three forms of action have been proposed: the characterization of current practices, through studies with care providers, in relation to their prescribing habits; The creation of guidelines and protocols for the rational use of antimicrobials; And finally, the development of educational materials and strategies [21].

The study in question also assesses the various types of infection that arise with the onset of neutropenia. Studies, made since the 1980s, have shown a decrease in the frequency of febrile episodes and infection with administration of prophylactic antibiotics. In hospital infection, like any infection acquired or transmitted in hospital space, it emerged in the medieval period, when institutions were set up to house sick people, pilgrims, the poor and the disabled, including places of separation and exclusion [22].

Of course, the indiscriminate gathering of people in a confined environment facilitated the transmission of contagious diseases, and the origin of the hospital infection could be traced back to that period. The first practices of infection control came only with the transformation of the hospital, from a place of care to the poor; where people were hospitalized even to die, to a place of healing and medicalization, from the eighteenth century. With the advance of the knowledge about the biological body and the consequent invasive interventions represented mainly by the surgeries, the control of the medium was not enough to avoid a new form of infection that began to emerge from these procedures. Among this knowledge, we highlight bacteriology and its consequent applications: asepsis, antisepsis, disinfection, antibiotic therapy and sterilization not only of the environment but also of man. Procedures used not only in a therapeutic way, but as prophylaxis of infections that could occur [22].

Despite the development of all the devices to control hospital infections, they continue to appear with resistant microorganisms, requiring preventive measures and collective control for a clinical practice that has always been curative and individual, the organization of a work process peculiarity through the creation of hospital infection control commissions in each hospital, choosing epidemiological surveillance as its main instrument, the multidisciplinary character of the agents of this work process, emphasizing the nurse as an indispensable professional and the multi-causality for the occurrence of these infections, Determining the need to establish risk factors [15].

Through Table 1 and Table 2, it is possible to observe antimicrobials resistant and sensitive to Gram-negative bacteria. Where the drugs: Lomefloxacin, Chlorphenicol, Ampicillin and Oxacillin appeared in higher percentages for resistance to Gram-negative and Imipenem, Linezolide, Gentamicin and Ticarcilna appeared in greater percentages for sensitivity to Gram-negative.

In Table 3 and Table 4, it is possible to observe antimicrobials resistant and sensitive to positive bacteria. Where the drugs: Penicillin, Oxacillin, Clindamycin and Ciprofloxacin appeared in higher percentages for resistance to Gram-positive and Vancomycin, Linezolid, Rifampicin, Imipenem and Amicacin appeared in greater percentages for sensitivity to Gram-positive. 
Table 1. Antimicrobial resistance profile a Gram negative microorganisms.

\begin{tabular}{ccc}
\hline Medicament & N & Percentual \\
\hline Lomefloxacin & 6 & $40 \%$ \\
Clorafenicol & 6 & $40 \%$ \\
Ampicilin & 6 & $40 \%$ \\
Oxacilin & 6 & $40 \%$ \\
Sulbactam & 3 & $20 \%$ \\
Norfloxacin & 3 & $20 \%$ \\
Amp + Sulbactan & 2 & $13 \%$ \\
Ceftazidin & 2 & $13 \%$ \\
Tobramicin & 2 & $13 \%$ \\
Piper + Tazo & 2 & $13 \%$ \\
Sulfa + Trimetr & 1 & $6 \%$ \\
Eritromicin & 1 & $6 \%$ \\
Vancomicin & 1 & $6 \%$ \\
Rifampicin & 1 & $6 \%$ \\
Ciprofloxacin & 1 & $6 \%$
\end{tabular}

Table 2. Antimicrobial susceptibility profile to Gram negative microorganisms.

\begin{tabular}{ccc}
\hline Medication & N & Percentage \\
\hline Mipenem & 6 & $40 \%$ \\
Linezolid & 6 & $40 \%$ \\
Ticarcillin & 4 & $27 \%$ \\
Gentamicin & 3 & $20 \%$ \\
Tobramycin & 2 & $13 \%$ \\
Ceftazidime & 2 & $13 \%$ \\
Tobramycin & 2 & $13 \%$ \\
Nitrofurantoin & 2 & $13 \%$ \\
Ampicilin & 1 & $6 \%$ \\
Choramphenicol & 1 & $6 \%$ \\
Levofloxacin & 1 & $6 \%$ \\
Piper + Tazo & 1 & $6 \%$ \\
Sulfa + Trimetr & 1 & $6 \%$ \\
Teicoplamin & 1 & $6 \%$ \\
\hline
\end{tabular}


Table 3. Antimicrobial resistance profile a Gram positive microorganisms.

\begin{tabular}{ccc}
\hline Medication & N & Percentage \\
\hline Penicillin & 8 & $53 \%$ \\
Oxacillin & 7 & $47 \%$ \\
Clindamycine & 5 & $33 \%$ \\
Ciprofloxacin & 5 & $33 \%$ \\
Clorafenicol & 4 & $27 \%$ \\
Norfloxacin & 4 & $27 \%$ \\
Sulfa + Trimet & 3 & $20 \%$ \\
Erythromycin & 2 & $13 \%$ \\
Nitrofuratoína & 2 & $13 \%$ \\
Gentamicin & 2 & $13 \%$ \\
Novabiocin & 1 & $6 \%$ \\
Linezolid & 1 & $6 \%$ \\
Teicoplamin & 1 & $6 \%$ \\
Rifampicin & 1 & $6 \%$ \\
Levofloxacin & 1 & \\
\hline & & $6 \%$ \\
\hline
\end{tabular}

Table 4. Sensitivity profile of antimicrobials a Gram positive microorganisms.

\begin{tabular}{ccc}
\hline Medication & N & Percentage \\
\hline Vancomycin & 8 & $53 \%$ \\
Linezolid & 7 & $47 \%$ \\
Penicillin & 6 & $33 \%$ \\
Rifampicin & 6 & $33 \%$ \\
Imipenem & 6 & $33 \%$ \\
Amikacin & 6 & $33 \%$ \\
Oxacillin & 4 & $27 \%$ \\
Clorafenicol & 4 & $8 \%$ \\
Ampicillin & 4 & $27 \%$ \\
Amox + acclavu & 3 & $20 \%$ \\
Novabiocin & 3 & $20 \%$ \\
Erythromycin & 3 & $20 \%$ \\
Clindamycine & 3 & $6 \%$ \\
Ofloxacin & 2 & $6 \%$ \\
Lomefloxacin & 2 & \\
\hline
\end{tabular}

The tables can be explained by the fact that there are several distinct mechanisms by which microorganisms can exhibit resistance to drugs, they are: the production of enzymes by the microorganisms that inactivate the active drug, 
for example, Beta lactamases inactivates penicillins and cephalosporins hydrolyzing the beta ring of lactam drugs. Modification of the permeability of the drug by the bacterium, so that the intracellular concentration of a given drug is not reached; For example, tetracycline is less concentrated in resistant than susceptible bacteria. Synthesis of an altered structural target for which the drugs are ineffective; for example, a methylated $23 \mathrm{~S}$ rRNA results in resistance to erythromycin. Development of an altered metabolic pathway, which omits the inhibited reaction by the drug and elaboration of altered enzyme that still has the capacity to perform its metabolic function, but less affected by the drug. The source of drug resistance may be genetic or not.

Medicines are considered the main therapeutic tool for recovery or maintenance of the health conditions of the population. However, the symbolism that they are coated and, consequently, their use by society, have contributed to the emergence of many adverse events, with high impact on the health and costs of the systems. Thus, promoting the rational use of medicines is an important tool to act with society, otherwise eliminate and minimize the problem. In this sense, the pharmacist can contribute considerably, since this is subject pertinent to its field of action. Their participation in multidisciplinary teams adds value to services and contributes to health promotion [23].

With the practice of pharmaceutical care and the lack of the population of a pharmacist more active in defense of the rational use of medicines, a unique opportunity arises for the performance of its role in society. Part of its attributions is the promotion of health, mainly through the provision of a quality pharmacy service, health education, with easy access to the population. Irrational drug use is an important public health problem; Therefore, it is necessary to consider the potential contribution of the pharmacist and to effectively incorporate it into the health teams in order to guarantee the improvement of the use of medicines, reducing the risks of morbidity and mortality, and that their work provides the means for the related costs Pharmacotherapy are as small as possible for society [24].

Infectious complications in cancer patients are usually severe and potentially fatal, thus justifying prevention and control measures. According to the Guideline Management of multidrug-resistant organisms in healthcare settings [7], the recommended interventions for the control of multiresistant microorganisms in the health services are grouped into seven categories: administrative support, careful use of antimicrobials, surveillance the use of standard and contact precautions, environmental and educational measures and decolonization [18].

The indiscriminate use of antibiotics can be addressed by establishing continuous or systematic forms of surveillance for the use of antimicrobials in the population as well as local microbial resistance standards or by developing and disseminating manuals on the rational prescription of antimicrobials based on efficacy assessments treatment and monitoring of local microbial resistance patterns and availability of rapid microbiological tests to guide therapeutics [25]. 


\section{Conclusions}

The results of this research show a high female prevalence among patients with Gram positive microorganisms, and the same proportion in patients with Gram negative microorganisms. The distribution of microorganisms with predominance of Gram positive bacteria in $69 \%$ of the patients, and only $31 \%$ of gram negative bacteria can be evaluated.

Of the patients analyzed, with a Gram-negative microbial profile, $75 \%$ had Escherichia coli infection and 25\% had Acinetobacter sp. Infection. In the analyzed patients, with a Gram-positive microbial profile, $56 \%$ presented infection by Sthapylococcus epidermidis, 22\% Sthapylococcus aureus, 16\% Enterococcus and $5 \%$ Rodhococcus.

Excessive use of antimicrobials in hospitalized patients leads to the suppression of drug-sensitive microorganisms from the intestinal flora and promotes the persistence and growth of resistant bacteria. The emergence of drug resistance in infections can be minimized by maintaining high levels of the drug in the tissues enough to inhibit both the original population and the first mutants while simultaneously administering two drugs that do not exhibit cross-resistance, delaying the emergence of resistant mutants. Exposure of microorganisms to the drug should be avoided, limiting its administration, especially in hospitals that have closed sites, favoring the transmission of microorganisms through the hospital staff, fomites and by direct contact.

The use of antibiotics in a curative way in most cases will be done in conjunction with others with other therapeutic measures. The determination of the existence of bacterial infection, however, is the fundamental requirement to indicate the antibiotic in a curative way. In some cases, there is no time to wait for the result of antibiograms, so it is necessary to perform the Gram test that provided important information for the beginning of an empirical antibiotic therapy. Knowledge of the hospital's bacterial profile may provide valuable insight into the clinical handling of the case.

However, the use of sterilization devices, disinfectants and antiseptics in thermosensitive instruments of hospitals, skin and mucosa of health professionals is indispensable for the control of the dissemination of bacteria and the role of the pharmacist is fundamental for the promotion, especially in the rational use of antibiotics, since in addition to monitoring and verifying the medical prescription it evaluates the adverse effects and acts in the orientation of how the medicine should be used.

\section{Acknowledgements}

To the Hospital do Câncer de Muriaé-Fundação Cristiano Varella, Centro de Ensino Superior de Valença-Fundação Dom André Arcoverde and to all those who contributed directly or indirectly to this study.

\section{References}

[1] Andrade, M. and Silva, S.R. (2007) Chemotherapy Administration: A Proposal of 
Nursing Protocol. Revista Brasileira de Enfermagem, 60, 331-335. https://doi.org/10.1590/S0034-71672007000300016

[2] Trufelli, D.C., Miranda, V.C., Santos, M.B.B., Fraile, N.M.P., Pecoroni, P.G., Gonzaga, S.F.R., Riechemann, R., Kaliks, R. and Del Giglio, A. (2008) Analysis of Delayed Diagnosis and Treatment of Breast Cancer in a Public Hospital. Journal of the Brazilian Medical Association, 54, 72-76.

[3] Beltrão, M.R.L.R., Vasconcelos, M.G.L., Bridges, C.M. and Albuquerque, M.C. (2007) Childhood Cancer: Maternal Perceptions and Strategies of Coping with the Diagnosis. Jornal de Pediatria (Rio ), 83, 562-566.

https://doi.org/10.2223/JPED.1723

[4] Medeiros, S.F., Maitelli, A. and Nince, A.P.B. (2007) Effects of Menopausal Hormone Therapy on the Immune System. Brazilian Journal of Gynecology and Obstetrics, 29, 593-601.

[5] Pietszkowski, N.C., Carvalho, G.A., Souza, H.N., Werka, C.R., Borazo, L.A., Graf, H. and Carvalho, M. (2007) Positive Anti-Neutrophil Cytoplasmic Antibody (ANC) Induced by Propylthiouracil: Case Report and Literature Review. Endocrinology and Metabolism, 51, 136-141.

[6] Abrantes, P.M., Magalhães, S.M.S., Acúrcio, F.A. and Sakurai, E. (2008) The Quality of Antimicrobial Prescription in Public Outpatient Clinics of the Municipal Health Department of Belo Horizonte, MG. Ciênc Collective Health, 13, 711-720.

[7] Bezerra, I.C.C., Anominondas, T.C.L., Sisenando, H.A.A.A.C.N., Sisenando, S.T.L.C.N. and Moreira, F.S.M. (2007) Evaluation of Initial Empirical Antimicrobial Therapy in Cancer Patients with Febrile Neutropenia. Revista Infarma of the Regional Council of Pharmacy, 19, 114-116.

[8] Murphy, T.K. and Husted, D.S. (2004) Autoimmunity in Obsessive-Compulsive Disorder and Tic Disorders. Revista Brasileira de Psiquiatria, 26, 218-219. https://doi.org/10.1590/S1516-44462004000400001

[9] Murahovschi, J. (2003) The Child with Fever in the Office. Jornal de Pediatria (Rio ), 79, 55-64. https://doi.org/10.2223/JPED.1000

[10] Hinrichein, S.L. (2005) Infectious and Parasitic Diseases. Guanabara Koogan, Rio de Janeiro.

[11] Mendes, G.B. (2008) Rational Use of Drugs: The Fundamental Role of the Pharmacist. Ciênc Collective Health, 13, 569-571.

[12] Nicolini, P., Birth, J.W.L., Greco, K.V. and Menezes, F.G. (2008) Factors Related to the Medical Prescription of Antibiotics in Public Pharmacy in the Western Region of the City of São Paulo. Ciênc Collective Health, 13, 689-696.

[13] Brooks, G.F., Carroll, K.C., Butel, J.S., Morse, S.A. and Mietzner, T.A. (2014) Medical Microbiology from Jawetz, Melnick \& Adelberg. 26 ed. Artmed.

[14] Nascimento-Carvalho, C.M. (2006) Outpatient Antibiotic Therapy as a Factor of Induction of Bacterial Resistance: A Rational Approach for Airway Infections. Jornal de Pediatria (Rio )), 82, 146-152. https://doi.org/10.2223/JPED.1548

[15] Ferreira, J.B., Rapoport, P.B., Sakano, E., Kos, A.O.A., Piltcher, O.B., Pignatari, S.S.N., Pinheiro, S.D. and Mocellin, M. (2006) Efficacy and Safety of Sultamicillin (Ampicillin/Sulbactam) and Amoxicillin/Clavulanate in the Treatment of Upper Airway Infections in Adults: A Multicentre Open-Label Randomized Trial. Revista Brasileira de Otorrinolaringologia, 72, 104-111. https://doi.org/10.1590/S0034-72992006000100016 
[16] Grillo, V.T.R.S., Gonçalves, T.G., Júnior, J.C., Paniágua, N.C. and Teles, C.B.G. (2013) Bacterial Incidence and Antimicrobial Resistance Profile in Pediatric Patients of a Public Hospital in Rondônia, Brazil. Revista de Ciências Farmacêuticas Básica e Aplicada, 34, 117-123.

[17] Lima, M.E., Andrade, D. and Haas, V.J. (2007) Prospective Evaluation of the Occurrence of Infection in Critically Ill Patients of the Intensive Care Unit. Revista Brasileira De Terapia Intensiva, 19, 342-347. https://doi.org/10.1590/S0103-507X2007000300013

[18] Cataneo, C., Canini, S.R.M.S., Castro, P.T.O., Hayashida, M. and Gir, E. (2011) Evaluation of the Sensitivity and Specificity of the Criteria for the Isolation of $\mathrm{Pa}$ tients Admitted to a Hospital Specialized in Oncology. Revista Latino-Americana de Enfermagem, 19, 1072-1079. https://doi.org/10.1590/S0104-11692011000500003

[19] Carlucci, V.D.S., Braga, F.T.M.M., Kings, P.E.D. and Silveira, R.C.C.P. (2016) Nursing Care to Onco-Hematologic Patients Submitted to High Doses of Chemotherapy: Integrative Review. Revista de Enfermagem UFPE on Line, 10, 1544-1555.

[20] Oletto, K.Q. and Reis, C. (2005) Antimicrobial Susceptibility of Uropathogens in Outpatients in the City of Goiânia, GO. Journal of the Brazilian Society of Tropical Medicine, 38, 416-420.

[21] Berquó, L.S., Barros, A.J.D., Lima, R.C. and Bertoldi, A.D. (2004) Use of Antimicrobials in an Urban Population. Revista de Saúde Pública, 38, 239-246. https://doi.org/10.1590/S0034-89102004000200013

[22] Lacerda, R.A. and Egry, E.Y. (1997) Hospital Infections and Their Relation with the Development of Hospital Care: Reflections to Analyze Their Current Practices of Control. Revista Latino-Americana de Enfermagem, 5, 13-23.

[23] Ardes, P.S.D. (2002) The Irrational Use of Drugs and Pharmacovigilance in Brazil. Cad Public Health, 18, 1478-1479.

[24] Mota, D.M., Silva, M.G.C., Sudo, E.C. and Ortún, V. (2008) Rational Use of Medicines: An Economic Approach to Decision Making. Science \& Collective Health, 13, 589-601.

[25] Bergsten-Mendes, G. (2008) Rational Use of Drugs: The Fundamental Role of the Pharmacist. Ciênc Collective Health, 13, 569-571. 